\title{
A Thermistor Anemometer for Low-Flow-Rate Measurements
}

\author{
Hiroyuki Fujita, Tadahiko Ohhashi, Masahiro Asakura, Mitsuhiro Yamada, and Kenzo Watanabe, Fellow, IEEE
}

\begin{abstract}
A digital anemometer using a self-heated thermistor as a probe has been developed for low-flow-rate measurements. To enhance the probe sensitivity to flow rate while reducing convection due to self-heating to a minimum, the thermistor is kept at the lowest possible temperature for exhibiting the negative resistance. The heat removed from the thermistor is analyzed and compared with the measured results, to derive the characteristic function which relates the voltage across the thermistor to the flow rate and temperature. This function is transformed from the flow-rate domain to the time domain by the digital waveform synthesis. The carrier signal thus generated is pulse-width-modulated by the output of the probe to provide the digital representation of the flow rate under measurement. The cross-sensitivity of the probe to the fluid temperature is compensated by scaling the carrier amplitude. This process of quantization including linearization and compensation functions makes a high-accuracy, flow-rate measurement possible with a simple configuration.
\end{abstract}

\section{INTRODUCTION}

A IR FLOW measurement is very important for monitoring and controlling living and working environments in a building. For such purposes, the hot-wire anemometry is bestsuited because it can be used to measure turbulent flow in open space [1]. The principle of measurement is based on the resistance change of a hot-wire when exposed to a flow. The resistance change depends not only on the flow rate but also on the fluid temperature [2]. To reduce this cross-sensitivity, a wire is usually kept at the highest allowable temperature [3]. This causes another problem; the convection due to the hot wire disturbs the flow field to be measured. This makes it difficult to measure the low flow rate accurately.

A straightforward way of reducing the disturbance is to lower the operating temperature of a hot wire. Based on this idea, the behavior of a hot wire under the lower temperature operation is first investigated. For the hot wire, a miniature thermistor self-heated at the constant temperature is used because it is much cheaper and has much higher sensitivity than the Wollaston wire [4]. The thermistor anemometer operating in the constant temperature mode poses two difficulties to its signal processing. One is the compensation for the fluid temperature [5], and the other is linearization of highly nonlinear responses. These difficulties are overcome by finding out the characteristic function which describes the voltage across the thermistor as a function of flow rate

Manuscript received May 9, 1994; revised January 19, 1995.

H. Fujita, T. Ohhashi, and M. Asakura are with the Development Laboratory, Kurabe Industrial Corp., 4830 Takatsuka, Hamamatsu 432, Japan.

M. Yamada and K. Watanabe are with the Research Institute of Electronics, Shizuoka University, 3-5-1 Johoku, Hamamatsu 432, Japan.

IEEE Log Number 9411465.

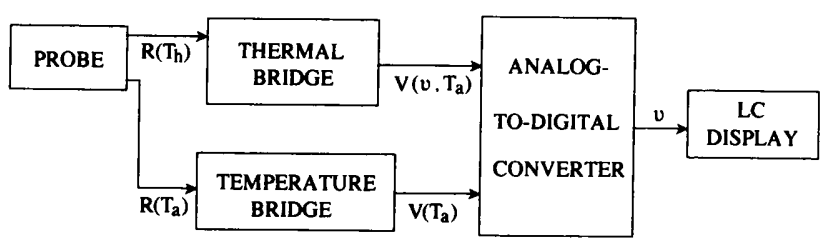

Fig. 1. A block diagram of the anemometer.

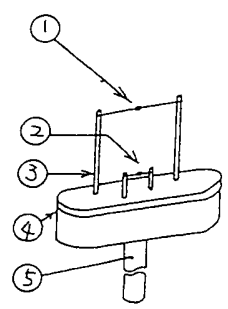

(1) Hot Thermistor

(2) Cold Thermistor

(3) Stainless steel stay

(4) Nylon base

(5) Connecting cable

Fig. 2. The configuration of the sensing probe.

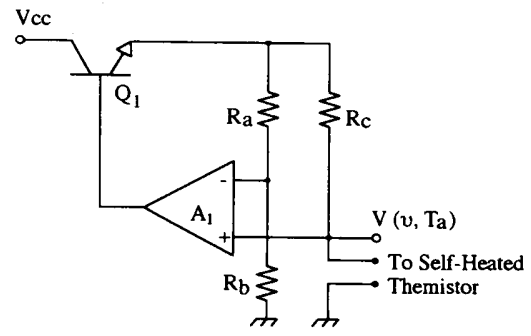

Fig. 3. The circuit diagram of the thermal bridge.

and temperature. The configuration and the operation of the anemometer based on the characteristic function will be described in the following sections.

\section{CONFIGURATION}

Fig. 1 shows a block diagram of the anemometer. It consists of the thermistor probe exposed to a cross flow, the thermal bridge to detect the flow rate, the temperature bridge to detect the fluid temperature, the analog-to-digital (A/D) converter to extract the flow rate from the output voltage of the thermal bridge, and the liquid crystal display (LCD) to indicate the flow rate and temperature in three-digit form.

The configuration of the probe is shown in Fig. 2. It is composed of two matched miniature thermistors welded to stainless steel stays [6]. The thermistors and stays are arranged such that they may not introduce any significant turbulence into the flow field to be measured. The thermistor suspended by long lead wires is connected to one arm of the Wheatstone bridge in the thermal bridge shown in Fig. 3 and is self-heated to $343 \mathrm{~K}$ for flow-rate measurements. This is much lower with respect to a conventional anemometer and is the lowest possible temperature for the thermistor to exhibit the negative resistance. The other thermistor detects the fluid temperature. 


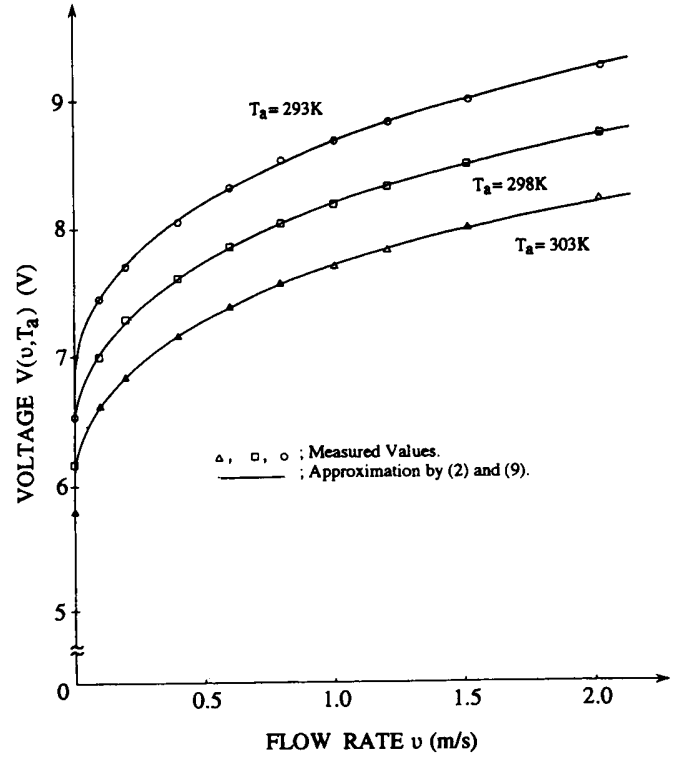

Fig. 4. The voltage across the self-heated thermistor as a function of the flow rate $v$ with the fluid temperature $T_{a}$ as a parameter.

The voltage $V\left(v, T_{a}\right)$ across the self-heated thermistor was measured as a function of flow rate $v$ and temperature $T_{a}$. The results are plotted in Fig. 4. Replotting data as a function of $T_{a}$ with $v$ being a parameter, one notices that $V\left(v, T_{a}\right)$ decreases linearly with the fluid temperature $T_{a}$ for a given flow rate. Therefore, $V\left(v, T_{a}\right)$ can be expressed as

$$
\begin{aligned}
V\left(v, T_{a}\right) & =V\left(v, T_{r}\right) f\left(T_{a}\right) \\
& =\left\{V\left(0, T_{r}\right)+\Delta V\left(v, T_{r}\right)\right\} f\left(T_{a}\right) \\
& =V\left(0, T_{r}\right)\left\{1+h\left(v, T_{r}\right)\right\} f\left(T_{a}\right)
\end{aligned}
$$

where $V\left(0, T_{r}\right)$ and $\Delta V\left(v, T_{r}\right)$ denote the offset and unbalance components, respectively, at some reference temperature $T_{r}$, and $h\left(v, T_{r}\right)$ is the characteristic function defined by $\Delta V\left(v, T_{r}\right) / V\left(0, T_{r}\right)$. The temperature dependence $f\left(T_{a}\right)$ is given by

$$
f\left(T_{a}\right)=1-0.012\left(T_{a}-298\right)
$$

This linear function fits the measured temperature dependence quite well; the correlation coefficient between them is larger than 0.99996, and the variance is smaller than 0.003 in the measured flow-rate range. A kernel of the characteristic function can be derived as follows: The heat dissipated by the thermistor per unit time is given by

$$
\begin{aligned}
\Phi & =C(v)\left(T_{h}-T_{a}\right) \\
& =C_{o}\left(1+k v^{1 / 2}\right)\left(T_{h}-T_{a}\right)
\end{aligned}
$$

where $C(v)=C_{o}\left(1+k v^{1 / 2}\right)$ is the thermal radiation coefficient at the flow rate $v, T_{h}$ is the thermistor temperature, and $k$ is a constant which depends on the heat conductance, the density, the specific heat of the fluid, and the dimensions of the thermistor. In thermal equilibrium, the heat loss of the thermistor is equal to the heating power, viz.,

$$
\frac{V^{2}\left(v, T_{a}\right)}{R\left(T_{h}\right)}=C_{o}\left(1+k v^{1 / 2}\right)\left(T_{h}-T_{a}\right)
$$

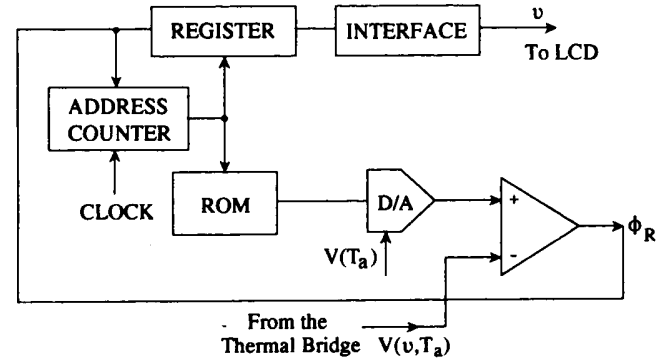

Fig. 5. The block diagram of the A/D converter.

where $R\left(T_{h}\right)$ is the thermistor resistance kept constant by the thermal bridge. Substituting (1) into (4) and noting that

$$
\frac{V^{2}\left(0, T_{a}\right)}{R\left(T_{h}\right)} f^{2}\left(T_{a}\right)=C_{o}\left(T_{h}-T_{a}\right)
$$

we get

$$
1+h\left(v, T_{r}\right)=\left(1+k v^{1 / 2}\right)^{1 / 2} .
$$

This indicates that a kernel of the characteristic function is $v^{1 / 2}$ for $k v^{1 / 2} \ll 1$, or $v^{1 / 4}$ for $k v^{1 / 2} \gg 1$. The former is amenable to the convection due to the self-heated thermistor. Thus the latter is adopted for further analysis.

The approximation using the kernel $v^{1 / 4}$ degrades in the low-flow-rate region because of the convection and the deviation from $v^{1 / 2}$. To compensate for these errors, a function which is sensitive to small $v$ is introduced:

$$
\phi(v)=\exp \left(\frac{\beta v+\gamma}{v+\alpha}\right)
$$

The constants $\alpha, \beta$, and $\gamma$ were determined such that the cost function $\Psi(\alpha, \beta, \gamma)$ defined by

$$
\Psi(\alpha, \beta, \gamma)=\frac{\text { variance }}{(\text { correlation coefficient })^{2}}=\frac{\sigma^{2}}{\rho^{2}}
$$

would be minimum. From this evaluation, $\alpha=0.32, \beta=$ 0.824 , and $\gamma=0.096$ are obtained. Solid lines in Fig. 4 show $V\left(v, T_{a}\right)$ calculated using the characteristic function

$$
h\left(v, T_{r}\right)=v^{1 / 4} \exp \left(\frac{0.824 v+0.096}{v+0.32}\right)
$$

and $f\left(T_{a}\right)$ given by (2). They agree quite well with the measured voltages. The standard deviation is smaller than 0.008 , and the correlation coefficient is 0.996 .

Fig. 5 shows the block diagram of the A/D converter. The ROM stores a priori the characteristic function $1+h\left(v, T_{r}\right)$ in table form with the flow rate $v$ being the address. Accessed sequentially by the incremental address counter, it sends the table data to the multiplying digital-to-analog (D/A) converter. The reference voltage of the D/A converter is the output voltage of the temperature bridge. Consisting of the Wheatstone bridge with one arm connected to the cold thermistor in Fig. 2 followed by the differential amplifier, the temperature bridge produces the output voltage $V\left(T_{a}\right)=V\left(0, T_{r}\right) f\left(T_{a}\right)$ which is consistent with the temperature-dependent offset component in (1). The D/A converter thus produces the carrier signal $v(t)$ given by

$$
v(t)=V\left(0, T_{r}\right)\left\{1+h\left(D, T_{r}\right)\right\} f\left(T_{a}\right)
$$



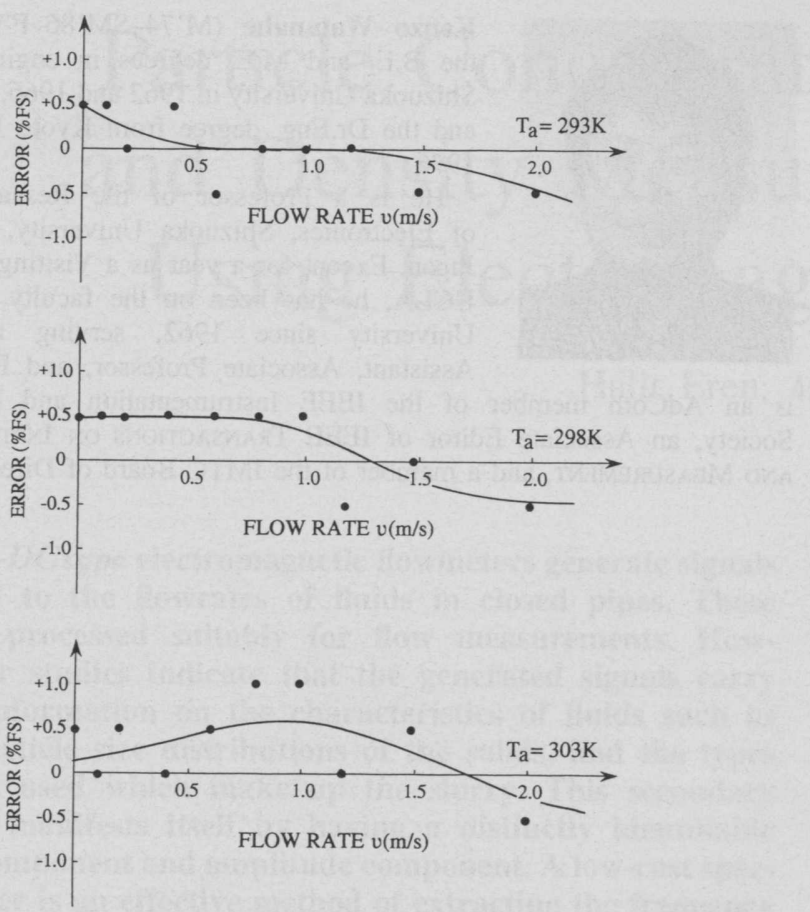

Fig. 6. Errors between readings of the prototype anemometer and manometer.

where $D$ is the table address. The comparator compares $v(t)$ with the output voltage $V\left(v, T_{a}\right)$ of the thermal bridge and latches the address $D$ when both the signals coincide. The address $D$ is then the digital representation of the flow rate under measurement, which is displayed on the LCD via the parallel interface.

The extraction of $v$ from $V\left(v, T_{a}\right)$ is possible also with the ratiometric $\mathrm{A} / \mathrm{D}$ conversion and the table look-up. Compared to the present method based on the direct digital waveform synthesis and the PWM, this alternative requires high-cost, high-precision components and the larger table because the characteristic function is nonlinear.

\section{PROTOTYPE ANEMOMETER}

The anemometer in Fig. 1 was built using off-the-shelf components, excepting the probe. For easy assembly, all the digital functions required for the $A / D$ conversion were implemented by a four-bit, one-chip microcomputer. It contains $256 \times$ four-bit RAM and $4 \mathrm{~K} \times 8$-bit ROM.

The resolution specified in the flow-rate range from 0 to $2 \mathrm{~m} / \mathrm{s}$ is $0.01 \mathrm{~m} / \mathrm{s}$. Thus, eight bits are enough for the table address. To meet the specified resolution, the characteristic function to be stored into the ROM should be two bits finer than the address because it approaches $v^{1 / 4}$ in the upper bound. For better accuracy, it was quantized into 12-bit words. Since one word occupies two bytes, 400 bytes of the ROM were allotted to the table. The table content is sent to the 12 bit multiplying D/A converter via three 4-bit ports. The time required to scan the table is $150 \mathrm{~ms}$. Another $50 \mathrm{~ms}$ is required for the one-chip microcomputer to process and display the flow rate. The sampling rate is thus five samples per second (sps).

To evaluate its performance, the prototype anemometer was applied to flow-rate measurements in a wind tunnel. The flow rate displayed on the LCD was compared with that measured by a commercial manometer. As plotted in Fig. 6 , relative errors between them are within $1 \%$ full scale (FS). Errors due to the fluid temperature are also within the specified resolution. These results confirm that the signal-processing techniques based on the characteristic function are valid for low-flow-rate measurements.

\section{CONCLUSIONS}

A digital anemometer was described which used a thermistor operating in the constant temperature mode. It allows flowrate measurements with a resolution of $0.01 \mathrm{~m} / \mathrm{s}$. Essential to this high resolution is the derivation of the characteristic function which describes the convection-dependent behavior of the self-heated thermistor. Another key technology which enables the low-cost implementation is the PWM based on the digital waveform synthesis. The high performance realized by these techniques makes the present anemometer attractive for environment monitoring and air flow control of fans and air conditioners. To exploit its performance in wider flow-rate and temperature range is a future work.

\section{REFERENCES}

[1] A. E. Perry, Hot-Wire Anemometry. Oxford: Clarendon Press, 1982.

[2] A. Boros, Electrical Measurements in Engineering. Amsterdam: Elsevier, 1985, chaps. 6 and 9.

[3] L. V. King, "On the convection of heat from small cylinders in a stream of fluid: Determination of the convection constants of small platinum wires with application to hot-wire anemometry," in Proc. Royal Soc., London, UK, 1914, vol. 90, pp. 563-570.

[4] K. Okamoto, T. Ohhashi, M. Asakura, and K. Watanabe, "A digital anemometer," IEEE Trans. Instrum. Meas., vol. 44, no. 2, pp. 116-120, 1995.

[5] A. Catellani, R. Stacchietti, A. Taroni, and C. Canali, "Performance and temperature stability of an air mass flowmeter based on a self-heated thermistor," Sensors Actuators, vol. 3, pp. 23-30, 1982/1983.

[6] "Small NTC thermistors; S series," Kurabe Tech. Note, catalog no. $\mathrm{N}-148$.

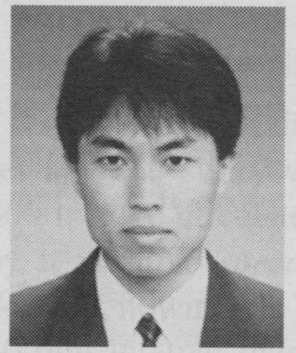

Hiroyuki Fujita received the B.Eng. and M.Eng. degrees from Shizuoka University, Hamamatsu, Japan, in 1991 and 1993, respectively.

In 1993, he joined Kurabe Industrial Co. Ltd. where he is now engaged in the development of measurement systems using thermistors.

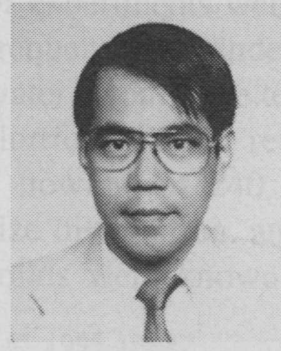

Tadahiko Ohhashi was born in Hamamatsu, Japan, on Jan. 6, 1958. He received the B.Eng. degree from Niigata University, Niigata, Japan, in 1980.

He joined Kurabe Industrial Co., Ltd., Hamamatsu, Japan, in 1980, and is now engaged in research and development of measuring equipment. 


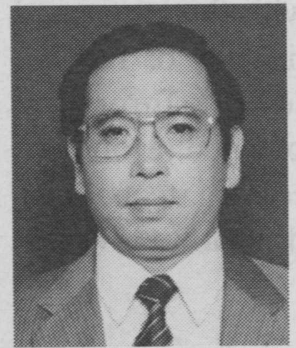

Masahiro Asakura received the B.Eng. degree from Gunma University, Gunma, Japan, in 1967. Upon graduation, he joined NEC, where he was engaged in the development of semiconductor power devices. In 1975, he moved to Shibaura Electric Co., Ltd., where he was engaged in the development of temperature and humidity detectors. In 1984, he joined Kurabe Industrial Co., Ltd., where he is managing the Development Laboratory.

Mitsuhiro Yamada received the B.E. and M.E. degrees in electronics engineering from Shizuoka University in 1990 and 1992, respectively. He is currently working toward the Dr.E. degree at Shizuoka University.

His research interests include sensor signal processing.

Mr. Yamada is a member of IEICE, Japan.

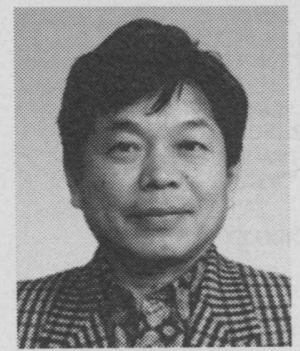

Kenzo Watanabe (M'74-SM'86-F'93) received the B.E. and M.E. degrees in engineering from Shizuoka University in 1962 and 1966, respectively, and the Dr.Eng. degree from Kyoto University in 1976.

$\mathrm{He}$ is a Professor of the Research Institute of Electronics, Shizuoka University, Hamamatsu, Japan. Except for a year as a Visiting Professor at UCLA, he has been on the faculty of Shizuoka University since 1962, serving as Research Assistant, Associate Professor, and Professor. He is an AdCom member of the IEEE Instrumentation and Measurement Society, an Associate Editor of IEEE TRANSACTIONS ON INSTRUMENTATION and MEASUREMENT, and a member of the IMTC Board of Directors. 